\title{
Neutron scattering reaps Nobel for physics
}

London. Techniques for probing the structure and properties of matter on the atomic scale have found much favour among the Nobel committee, from the Braggs' award for X-ray diffraction in 1915 to that for the scanning tunnelling microscope in 1986. This year's physics prize extends the same theme through its joint award to Bertrand Brockhouse and Clifford Shull for their work in developing neutron inelastic scattering and neutron diffraction respectively.

Both techniques rely on the scattering of neutrons by matter. Neutron scattering has never been the cheapest of experimental techniques. But the pervasive role of neutron scattering in condensed-matter physics can be justified on the grounds that it provides information that is otherwise inaccessible.

Neutron diffraction works in the same way as X-ray diffraction; the fact that the quantum-mechanical (de Broglie) wave-

\section{Chemist honoured for 'carbocations'}

London. The products of organic chemical reactions, such as ferrocene, dienes and crown ethers, have provided many Nobel prizes for chemistry in the past. This year, in contrast, the prize has been awarded for work on the transient species that come and go along the reaction pathway.

The Swedish Academy last week awarded the prize to Hungarian-born chemist George Olah for his studies of carbocations, positively charged species that are a common intermediate of many organic reactions.

Olah joined the scientific exodus from Hungary following the uprising of 1956 . He joined the Dow Chemicals Company in Canada in 1957 and is now director of the Loker Hydrocarbon Research Institute at the University of Southern California in Los Angeles.

There are two ways of turning a carbon-based molecule into a carbocation. One is to remove an

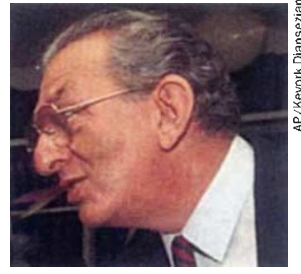

George Olah anion, leaving a carbenium ion with a formally three-coordinate carbon atom. The other is to add a hydrogen ion to a saturated carbon, creating a carbonium ion with a five-coordinate carbon.

In the 1920 s and 1930 s, C. K. Ingold proposed that carbenium ions are ephemeral reaction intermediates of certain elimination and substitution reactions of organic compounds, in which the first step is the loss of an anion. So common are these reactions that carbenium ions "dominate half of organic chemistry in terms of how things happen", according to Philip Eaton of the

\section{IMAGE UNAVAILABLE FOR COPYRIGHT REASONS}

\section{Shull: preparing to celebrate.}

length of thermal neutrons is of the same order as the wavelength of X-rays means they probe long-range order on the same length scales, which are those typical of atomic lattices. But neutron diffraction differs in important respects stemming from how neutrons interact with matter.

The neutron-scattering power of atoms depends in a very erratic way on the atomic

\section{University of Chicago.}

Carbonium ions, formed by protonation of alkyl carbons using superacids such as $\mathrm{HSO}_{3} \mathrm{~F}$ and $\mathrm{HF}_{-} \mathrm{SbF}_{5}$, are of particular interest because their bonding configuration cannot be rationalized according to classical charge is attached to an atom with a welldefined coordination number. Rather, these ions are 'non-classical' structures in which bonding is delocalized between several atoms, with the positive charge distributed among them.

In the days before ultrafast spectroscopic techniques, the lifetimes of these unstable species were too short for them to be observable. Working at Dow in the 1960s, Olah found a way to stabilize carbenium ions for long enough to use nuclear magnetic resonance and electron spectroscopy (ESCA) to elucidate their structure and properties.

Olah used the Lewis superacid $\mathrm{SbF}_{5}$ to extract halide ions from alkyl halides in solvents that were such poor nucleophiles that they did not readily attack the positively charged species. When he announced his success in analysing long-lived carbenium ions at a conference at Long Island in 1962 the news "hit like a bombshell" says Paul von Ragué Schleyer of the University of Erlangen-Nurnberg, Germany, who was there to hear it.

Schleyer points to the practical value of Olah's work as a major justification for the award. Protonation of hydrocarbons may lead to isomerization, for example converting straight-chain hydrocarbons into branched ones, which boosts the octane rating of hydrocarbon fuels. Such conversions are now commonly carried out using solid superacids such as zeolites. Philip Ball ideas of valence, under which the positive
\& mass. Neutron diffraction can therefore distinguish elements that are invisible or indistinguishable to $\mathrm{X}$-rays. Hydrogen in particular has a large neutron scattering crosssection, and neutrons can be used to find these light atoms in a crystal structure.

Adjacent elements in the periodic table are hard to differentiate with $\mathrm{X}$-rays, but can have vastly different neutron scattering crosssections. Neutron diffraction has therefore revealed the structure of metal alloys such as brass (a copper-zinc alloy). And even isotopes of the same element can look very different to neutrons. Furthermore, as neutrons have a magnetic moment, they can be scattered by magnetic atoms.

Working at Oak Ridge in Tennessee with the group of E. O. Wollan, Shull played a central role in the development of instrumentation for routine crystallographic analysis with neutrons. He was the first to demonstrate magnetic diffraction, from the compound manganese oxide. Before his retirement Shull was working at the Massachusetts Institute of Technology (MIT).

Collisions between neutrons and atoms can also be inelastic, leading to transfer of energy. Neutron scattering can therefore be used as a spectroscopic technique. In the 1950 s, Brockhouse developed the pioneering neutron spectrometer that forms the basis of instruments still in use today - an achievement that he carried off almost singlehandedly, according to Bob Birgeneau of the MIT.

Inelastic neutron scattering is now a mainstay of physicists studying the dynamics of condensed phases, such as the lattice vibrations of solids (known as phonons) and the molecular motions in adsorbed phases. Brockhouse was the first to measure the phonon dispersion curve of a solid, a spectrum of the lattice vibrational energies. $\mathrm{He}$ has now retired from the physics department of McMaster University in Canada.

The selection of Brockhouse and Shull as the founders of neutron scattering is "absolutely the right choice", says Jim Jorgensen of Argonne National Laboratories. "They did amazing things in the early days," he adds. Peter Day of the Royal Institution in the United Kingdom and ex-director of the Institut Laue-Langevin in Grenoble, France, Europe's premier neutron-scattering centre, says that "these are giants of the field".

Although Europeans have been wellserved for neutron studies, the US situation remains under a cloud. In particular, the future of the proposed \$2.7-billion advanced neutron source at Oak Ridge is uncertain. "We're in a sad state of disarray in neutron funding in the United States," says Jorgensen. Will the Nobel awards boost the status of neutron scattering in condensed-matter science? "I'm counting on it," says Birgeneau.

Philip Ball 